International Journal of Applied Mathematics

Volume 30 No. 2 2017, 131-150

ISSN: 1311-1728 (printed version); ISSN: 1314-8060 (on-line version)

doi: http://dx.doi.org/10.12732/ijam.v30i2.5

\title{
FRACTIONAL NOETHER'S THEOREM WITH CLASSICAL \\ AND GENERALIZED FRACTIONAL DERIVATIVE OPERATORS
}

\author{
Hong-Bin Zhang ${ }^{1}{ }^{\S}$, Hai-bo Chen ${ }^{2}$ \\ ${ }^{1,2}$ College of Mechanical and Electronic Engineering \\ Chaohu University \\ Hefei, 238000, People's Republic of CHINA
}

\begin{abstract}
In this paper, we present two new "transfer formulas" for some generalized fractional derivative operators, and derive a Noether type symmetry theorem to fractional problems of the calculus of variations with classical and generalized fractional derivative operator. As a result, we obtain constants of motion that are valid along Euler-Lagrange extremals for mixed classical and fractional derivatives. This theorem provides an explicit algorithmic way to compute constants of motion for Lagrangian systems with classical and generalized fractional derivative operator admitting a symmetry. Results from previous literature can be obtained as a special case of one.
\end{abstract}

AMS Subject Classification: 49K05, 49S05, 70H33

Key Words: calculus of variations, generalized fractional derivative operator, Euler-Lagrange equations, invariance, Noether's theorem

\section{Introduction}

Among the mathematical machinery of classical mechanics, the Noether theorem of calculus of variation became one of the most important theorems for physics in the 20th century. Since the seminal work of Emmy Noether [1] it was well know that all conservations laws in mechanics, e.g., conservation of energy or conservation of momentum, are directly related to the invariance of the action under a family of transformations.

Received: February 7, 2017

(C) 2017 Academic Publications

${ }^{\S}$ Correspondence author 
In 1931, Bauer [2] proved that it is impossible to use a variational principle to derive a single linear dissipative equation of motion with constant coefficients. The Bauer theorem expresses the well-known belief that there is no direct method of applying variational principles to nonconservative systems. Over the last century, several methods were developed in order to deal nonconservative systems, such as introducing time dependent Lagrangians and introducing auxiliary coordinates that describe the reverse-time system, etc., [3]. However, all these approaches give us non-physical Lagrangians in the sense they provide non-physical relations for the momentum and Hamiltonian of the system.

In 1996, Riewe observed that the second-order derivative terms in EulerLagrange equations are corresponding to the square of the first-order derivative times in Lagrangians, therefore Riewe presented that the first-order derivative terms should come from the half-order derivative terms in Lagrangians. In view of this, Riewe brought the nonconservative forces into Lagrangian and Hamiltonian with applying the tools of fractional calculus [4], [5]. The idea proposed by Riewe proved to be so novel and interesting that it transcended the problem that it was originally designed to solve and became an area of study in its own right. Nowadays, the fractional calculus of variations is being developed as a tool to study a wide variety of problems [6].

Agrawal [7] presented a heuristic approach to obtain differential equations of fractionally damped systems. Later, Agrawal [8]-[10] further presented generalized Euler-Lagrange equations for unconstrained and constrained fractional variational problems involving Riemann-Liouville, Caputo and Riesz fractional derivatives. Klimek presented a fractional sequential mechanics model with symmetric fractional derivatives [11] and stationary conservation laws for fractional differential equations with variable coefficients [12]. Dreisigmeyer and Young [13] presented nonconservative Lagrangian mechanics using a generalized function approach. In [14], the authors showed that obtaining differential equations for a nonconservative system using fractional variational calculus may not be possible. Cresson [15] presented fractional embedding of differential operators and Lagrangian systems where it was shown that the embedding procedure is compatible with fractional variational calculus. Cresson also developed several formulations for fractional mechanics.

The study on the symmetries and conserved quantities for fractional variational problems is an important and valuable aspect about fractional dynamics. In 2007, Frederico and Torres [16] firstly studied invariance properties of fractional variational problems with Riemann-Liouville derivatives, established the Noether theorem by introducing a new concept of fractional conserved quantity, and further extended it to the situation of the Caputo derivative [17] 
and Riesz-Caputo derivative [18]. Zhou and $\mathrm{Fu}[19]$ presented the fractional Noether's theorem of the Hamiltonian systems based on Frederico's definition of fractional conserved quantity. Considering this fractional conserved quantity is not constant in time, so the concept of conserved quantity is not clear, Atanacković [20] derived infinitesimal criterion for a local one-parameter group of transformations to be a variational symmetry group for the fractional variational problem, and further present a Noether type theorem in terms of conservation laws as it is done in the classical theory. Zhang and Zhai [21] studied Noether symmetries and conserved quantities for Birkhoffian systems based on the strategy used by Atanacković. Because the formula of conserved quantity contain an integral relations, the result is also unsatisfactory. In 2013, Bourdin [22] firstly proved a "transfer formula", and then using this "transfer formula", the fractional conserved quantity introduced by Frederico and Torres be converted into a new form conserved quantity. The formula of conserved quantity is algorithmic, it can be used to computer conserved quantity to arbitrary high order approximations. In 2010, Agrawal [23] introduced two new differential operators (generalized fractional derivatives operators) and defined a new class of variational problems, which in special cases reduce to fractional variational problems with the left and right fractional Riemann-Liouville, Caputo, Riesz, Riesz-Caputo derivatives, respectively.

In the present work, we will further develop the idea of Bourdin, and present a new "transfer formula" for generalized fractional derivatives operators introduced by Agrawal, and using this new "transfer formula" deduce generalize Noether's theorems for Lagrangian depending on mixed classical and generalized fractional derivative operators. When the parameters of generalized fractional derivatives operators $p$ and $l$ take different values, we can obtain Noether's theorems for Lagrangian depending on mixed classical and RiemannLiouville, Caputo, Riesz fractional derivatives, respectively.

The article is organized as follows: In Section 2, a brief summary of the definitions and properties of the generalized fractional integrals and derivatives are proposed. The main contributions of the paper appear in Section 3, we first derive fractional Euler-Lagrange equations and necessary condition of invariance, present a new "transfer formula", then prove the extension of Noether's theorem to fractional problems of the calculus of variations with classical and generalized fractional derivatives operators. Finally, a conclusion is given in Section 4. 


\section{Definition and Properties of Some Generalized Fractional Order Operators}

For convenience, we briefly overview definitions and properties of some generalized operators for integration and differentiation of frcational order.

First, consider a generalized integral operator $K_{P}^{\alpha}$ of order $\alpha$, which is defined as follows in [23].

Definition 1. (A generalized fractional integral) The operator $K_{P}^{\alpha}$ is given by

$$
K_{\left\langle t_{1}, t, t_{2}, p, q\right\rangle}^{\alpha} f(t)=p \int_{t_{1}}^{t} k_{\alpha}(t, \tau) f(\tau) d \tau+l \int_{t}^{t_{2}} k_{\alpha}(\tau, t) f(\tau) d \tau=K_{P}^{\alpha} f(t),
$$

where $t_{1}<t<t_{2}, P=\left\langle t_{1}, t, t_{2}, p, l\right\rangle$ is a parameter set, $k_{\alpha}(t, \tau)$ is a kernel which may depend on a parameter $\alpha$, and the parameters $p$ and $l$ are two real numbers.

Obviously, the new operator $K_{P}^{\alpha}$ satisfies the following properties:

Property 1. The operator $K_{P}^{\alpha}$ is a linear one, i.e. if $f_{1}(t)$ and $f_{2}(t)$ are two functions, then

$$
K_{P}^{\alpha}\left(f_{1}(t)+f_{2}(t)\right)=K_{P}^{\alpha} f_{1}(t)+K_{P}^{\alpha} f_{2}(t) .
$$

Property 2. The new operator $K_{P}^{\alpha}$ satisfies the following formulae

$$
K_{P}^{\alpha} f(t)=p K_{P_{1}}^{\alpha} f(t)+l K_{P_{2}}^{\alpha} f(t),
$$

where $P=\left\langle t_{1}, t, t_{2}, p, l\right\rangle, P_{1}=\left\langle t_{1}, t, t_{2}, 1,0\right\rangle$ and $P_{2}=\left\langle t_{1}, t, t_{2}, 0,1\right\rangle$.

Property 3. The operator $K_{P}^{\alpha}$ and $K_{P^{*}}^{\alpha}$ satisfies the following integration by parts formula

$$
\int_{t_{1}}^{t_{2}} g(t) K_{P}^{\alpha} f(t) d t=\int_{t_{1}}^{t_{2}} f(t) K_{P^{*}}^{\alpha} g(t) d t
$$

where $P=\left\langle t_{1}, t, t_{2}, p, l\right\rangle$ and its dual $P^{*}=\left\langle t_{1}, t, t_{2}, l, p\right\rangle$.

Second, consider two generalized derivative operators $A_{P}^{\alpha}$ and $B_{P}^{\alpha}$, which are defined as follows:

Definition 2. (A generalized Riemann-Liouville fractional derivative) The operator $A_{P}^{\alpha}$ is given by

$$
A_{\left\langle t_{1}, t, t_{2}, p, l\right\rangle}^{\alpha} f(t)=D^{n} K_{P}^{n-\alpha} f(t)=A_{P}^{\alpha} f(t),
$$

where $D$ is the classical derivative operator, $n-1<\alpha<n, n$ being an integer. We refer to $A_{P}^{\alpha}$ as operator $A$ of order $\alpha$ and $p$-set $P$. 
Definition 3. (A generalized Caputo fractional derivative) The operator $B_{P}^{\alpha}$ is given by

$$
B_{\left\langle t_{1}, t, t_{2}, p, l\right\rangle}^{\alpha} f(t)=K_{P}^{n-\alpha} D^{n} f(t)=B_{P}^{\alpha} f(t) .
$$

We refer to $B_{P}^{\alpha}$ as operator $B$ of order $\alpha$ and $p$-set $P$.

The operators $A_{P}^{\alpha}$ and $B_{P}^{\alpha}$ satisfy the following properties:

Property 4. The operators $A_{P}^{\alpha}$ and $B_{P}^{\alpha}$ are also linear, i.e. if $f_{1}(t)$ and $f_{2}(t)$ are two functions, then

$$
\begin{aligned}
& A_{P}^{\alpha}\left(f_{1}(t)+f_{2}(t)\right)=A_{P}^{\alpha} f_{1}(t)+A_{P}^{\alpha} f_{2}(t), \\
& B_{P}^{\alpha}\left(f_{1}(t)+f_{2}(t)\right)=B_{p}^{\alpha} f_{1}(t)+B_{P}^{\alpha} f_{2}(t) .
\end{aligned}
$$

Property 5. The operators $A_{P}^{\alpha}$ and $B_{P}^{\alpha}$ satisfy the following formulas for integration by parts,

$$
\begin{gathered}
\int_{t_{1}}^{t_{2}} g(t) A_{P}^{\alpha} f(t) d t=(-1)^{n} \int_{t_{1}}^{t_{2}} f(t) B_{P^{*}}^{\alpha} g(t) d t \\
+\left.\sum_{j=0}^{n-1}(-1)^{j} A_{P}^{\alpha+j-n} f(t) D^{n-1-j} g(t)\right|_{t_{1}} ^{t_{2}}, \\
\int_{t_{1}}^{t_{2}} g(t) B_{P}^{\alpha} f(t) d t=(-1)^{n} \int_{t_{1}}^{t_{2}} f(t) A_{P^{*}}^{\alpha} g(t) d t \\
+\left.\sum_{j=0}^{n-1}(-1)^{j} A_{P^{*}}^{\alpha+j-n} g(t) D^{n-1-j} f(t)\right|_{t_{1}} ^{t_{2}},
\end{gathered}
$$

where $f(t)$ and $g(t)$ are sufficiently smooth functions, and $n-1<\alpha<n$.

When $0<\alpha<1$, we have following integration relations:

$$
\begin{aligned}
& \int_{t_{1}}^{t_{2}} g(t) A_{P}^{\alpha} f(t) d t=\left.g(t) K_{P}^{1-\alpha} f(t)\right|_{t_{1}} ^{t_{2}}-\int_{t_{1}}^{t_{2}} f(t) B_{P^{*}}^{\alpha} g(t) d t, \\
& \int_{t_{1}}^{t_{2}} g(t) B_{P}^{\alpha} f(t) d t=\left.f(t) K_{P^{*}}^{1-\alpha} g(t)\right|_{t_{1}} ^{t_{2}}-\int_{t_{1}}^{t_{2}} f(t) A_{P^{*}}^{\alpha} g(t) d t .
\end{aligned}
$$

Property 6. Let $0<\alpha<1, P=\left\langle t_{1}, t, t_{2}, p, l\right\rangle$, and $f(t) \in A C\left(\left[t_{1}, t_{2}\right]\right)$, if the kernel $k_{1-\alpha}$ is integrable and there exist functions $h$ and $g$ such that

$$
\int_{a}^{t} k_{1-\alpha}(\theta, \tau) d \theta+\int_{a}^{\tau} k_{1-\alpha}(t, \theta) d \theta=g(t)+h(\tau)
$$

for all $t, \tau \in\left[t_{1}, t_{2}\right]$, then the following relation holds [18]:

$$
A_{P}^{\alpha} f(t)=p f\left(t_{1}\right) k_{1-\alpha}\left(t, t_{1}\right)-l f\left(t_{2}\right) k_{1-\alpha}\left(t_{2}, t\right)+B_{P}^{\alpha} f(t) .
$$


Remark. For new operators $A_{P}^{\alpha}$ and $B_{P}^{\alpha}$, we have the following relations

$$
\begin{gathered}
A_{P_{1}}^{\alpha}=A_{P_{2}^{*}}^{\alpha}, \quad A_{P_{2}}^{\alpha}=A_{P_{1}^{*}}^{\alpha}, \quad A_{P_{3}}^{\alpha}=A_{P_{3}^{*},}^{\alpha}, \\
B_{P_{1}}^{\alpha}=B_{P_{2}^{*}}^{\alpha}, \quad B_{P_{2}}^{\alpha}=B_{P_{1}^{*}}^{\alpha}, \quad B_{P_{3}}^{\alpha}=B_{P_{3}^{*}}^{\alpha},
\end{gathered}
$$

where $P_{1}=\left\langle t_{1}, t, t_{2}, 1,0\right\rangle, P_{2}=\left\langle t_{1}, t, t_{2}, 0,1\right\rangle$ and $P_{3}=\left\langle t_{1}, t, t_{2}, \frac{1}{2}, \frac{1}{2}\right\rangle$.

In particular, let $0<\alpha<1, k_{\alpha}(t, \tau)=\frac{1}{\Gamma(\alpha)}(t-\tau)^{\alpha-1}$. If $P=P_{1}=$ $\left\langle t_{1}, t, t_{2}, 1,0\right\rangle$, then

$$
K_{P_{1}}^{\alpha} f(t)=\frac{1}{\Gamma(\alpha)} \int_{t_{1}}^{t}(t-\tau)^{\alpha-1} f(\tau) d \tau={ }_{t_{1}} I_{t}^{\alpha} f(t)
$$

is a left Riemann-Liouville fractional integral of $f(t)$ of order $\alpha$,

$$
A_{P_{1}}^{\alpha} f(t)=\frac{1}{\Gamma(1-\alpha)} \frac{d}{d t} \int_{t_{1}}^{t}(t-\tau)^{-\alpha} f(\tau) d \tau={ }_{t_{1}} D_{t}^{\alpha} f(t)
$$

is a left Riemann-Liouville fractional derivative of $f(t)$ of order $\alpha$,

$$
B_{P_{1}}^{\alpha} f(t)=\frac{1}{\Gamma(1-\alpha)} \int_{t_{1}}^{t}(t-\tau)^{-\alpha} f^{\prime}(\tau) d \tau={ }_{t_{1}}^{C} D_{t}^{\alpha} f(t)
$$

is a left Caputo fractional derivative of $f(t)$ of order $\alpha$.

The relation between $t_{1} D_{t}^{\alpha} f(t)$ and ${ }_{t_{1}}^{C} D_{t}^{\alpha} f(t)$ is given by following formula

$$
{ }_{t_{1}}^{C} D_{t}^{\alpha} f(t)={ }_{t_{1}} D_{t}^{\alpha}\left(f-f\left(t_{1}\right)\right) \text {. }
$$

If $P=P_{2}=\left\langle t_{1}, t, t_{2}, 0,1\right\rangle$, then

$$
K_{P_{2}}^{\alpha} f(t)=\frac{1}{\Gamma(\alpha)} \int_{t}^{t_{2}}(\tau-t)^{\alpha-1} f(\tau) d \tau={ }_{t} I_{t_{2}}^{\alpha} f(t)
$$

is the right Riemann-Liouville fractional integral of $f(t)$ of order $\alpha$,

$$
-A_{P_{2}}^{\alpha} f(t)=-\frac{1}{\Gamma(1-\alpha)} \frac{d}{d t} \int_{t}^{t_{2}}(\tau-t)^{-\alpha} f(\tau) d \tau={ }_{t} D_{t_{2}}^{\alpha} f(t)
$$

is the right Riemann-Liouville fractional derivative of $f(t)$ of order $\alpha$,

$$
-B_{P_{2}}^{\alpha} f(t)=-\frac{1}{\Gamma(1-\alpha)} \int_{t}^{t_{2}}(\tau-t)^{-\alpha} f^{\prime}(\tau) d \tau={ }_{t}^{C} D_{t_{2}}^{\alpha} f(t)
$$

is the right Caputo fractional derivative of $f(t)$ of order $\alpha$.

The relation between ${ }_{t} D_{t_{2}}^{\alpha} f(t)$ and ${ }_{t}^{C} D_{t_{2}}^{\alpha} f(t)$ is given by following formula

$$
{ }_{t}^{C} D_{t_{2}}^{\alpha} f(t)={ }_{t} D_{t_{2}}^{\alpha}\left(f-f\left(t_{2}\right)\right) \text {. }
$$


If $P=P_{3}=\left\langle t_{1}, t, t_{2}, \frac{1}{2}, \frac{1}{2}\right\rangle$, then

$$
K_{P_{3}}^{\alpha} f(t)=\frac{1}{2}\left[K_{P_{1}}^{\alpha} f(t)+K_{P_{2}}^{\alpha} f(t)\right]=\frac{1}{2}\left[t_{1} I_{t}^{\alpha} f(t)+{ }_{t} I_{t_{2}}^{\alpha} f(t)\right]={ }_{t_{1}}^{R} I_{t_{2}}^{\alpha} f(t)
$$

is the Riesz fractional integral of $f(t)$ of order $\alpha$,

$$
A_{P_{3}}^{\alpha} f(t)=\frac{1}{2}\left[A_{P_{1}}^{\alpha} f(t)+A_{P_{2}}^{\alpha} f(t)\right]=\frac{1}{2}\left[t_{1} D_{t}^{\alpha} f(t)-{ }_{t} D_{t_{2}}^{\alpha} f(t)\right]={ }_{t_{1}}^{R} D_{t_{2}}^{\alpha} f(t)
$$

is the Riesz fractional derivatives of $f(t)$ of order $\alpha$,

$$
B_{P_{3}}^{\alpha} f(t)=\frac{1}{2}\left[B_{P_{1}}^{\alpha} f(t)+B_{P_{2}}^{\alpha} f(t)\right]=\frac{1}{2}\left[{ }_{t_{1}}^{C} D_{t}^{\alpha} f(t)-{ }_{t}^{C} D_{t_{2}}^{\alpha} f(t)\right]={ }_{t_{1}}^{R C} D_{t_{2}}^{\alpha} f(t)
$$

is the Riesz-Caputo fractional derivative of $f(t)$ of order $\alpha$.

The relation between ${ }_{t_{1}}^{R} D_{t_{2}}^{\alpha} f(t)$ and ${ }_{t_{1}}^{R C} D_{t_{2}}^{\alpha} f(t)$ is given by following formula

$$
{ }_{t_{1}}^{R C} D_{t_{2}}^{\alpha} f(t)={ }_{t_{1}}^{R} D_{t_{2}}^{\alpha} f(t)-\frac{1}{2}{ }_{t_{1}} D_{t}^{\alpha} f\left(t_{1}\right)+\frac{1}{2}{ }_{t} D_{t_{2}}^{\alpha} f\left(t_{2}\right) .
$$

For additional background on fractional calculus, we may refer to one of the many books on the subject, as [26]-[35].

\section{Main Results}

Firstly, we consider the extreme problem (of minimizing or maxi-minimizing) for the functional

$$
J_{A}(q)=\int_{t_{1}}^{t_{2}} L_{A}\left(t, q^{s}(t), \dot{q}^{s}(t), A_{P}^{\alpha} q^{s}(t)\right) d t
$$

subject to boundary conditions

$$
q^{s}\left(t_{1}\right)=q_{t_{1}}^{s}, \quad q^{s}\left(t_{2}\right)=q_{t_{2}}^{s},
$$

where $\left[t_{1}, t_{2}\right] \subset \mathbb{R}, t_{1}<t_{2}, 0<\alpha<1, s=1,2, \cdots, n$, the Lagrangian $L_{A}\left(t, q^{s}(t), \dot{q}^{s}(t), A_{P}^{\alpha} q^{s}(t)\right):\left(\left[t_{1}, t_{2}\right] \times \mathbb{R}^{n} \times \mathbb{R}^{n} \times \mathbb{R}^{n} ; \mathbb{R}\right)$ is assumed $C^{2}$-functions with respect to all its arguments, $q^{s}$ are generalized coordinates. We denote by $\partial_{i} L_{A}$ the partial derivative of $L_{A}$ with respect to its $i$ th argument, $i=1,2, \cdots, 5$.

Theorem 1. Let $q^{s}(t)$ be a solution to problem (27)-(28). Then $q^{s}(t)$ satisfies the generalized Euler-Lagrange equation 


$$
\frac{\partial L_{A}}{\partial q^{s}}-\frac{d}{d t} \frac{\partial L_{A}}{\partial \dot{q}^{s}}-B_{P^{*}}^{\alpha} \frac{\partial L_{A}}{\partial A_{P}^{\alpha} q^{s}}=0
$$

for $t \in\left[t_{1}, t_{2}\right]$.

Proof. Suppose that $q^{s}(t)$ is an extremizer of $J_{A}$. Consider the value of $J_{A}$ at a nearby admissible function $\bar{q}^{s}(t)=q^{s}(t)+\epsilon \xi^{s}(t)$, where $\epsilon \in \mathbb{R}$ is a small parameter and $\xi^{s}(t)$ is an arbitrary function satisfying $\xi^{s}\left(t_{1}\right)=\xi^{s}\left(t_{2}\right)=0$. A necessary condition for $q^{s}(t)$ to be an extremizer is given by $\left.\frac{d J_{A}(\epsilon)}{d \epsilon}\right|_{\epsilon}=0$, i.e.,

$$
\int_{t_{1}}^{t_{2}}\left(\frac{\partial L_{A}}{\partial q^{s}} \xi^{s}(t)+\frac{\partial L_{A}}{\partial \dot{q}^{s}} \dot{\xi}(t)+\frac{\partial L_{A}}{\partial A_{P}^{\alpha} q^{s}} A_{P}^{\alpha} \xi^{s}(t)\right) d t=0 .
$$

Using the classical integration by parts formula as well as formula (11), we obtain that

$$
\begin{gathered}
\int_{t_{1}}^{t_{2}} \frac{\partial L_{A}}{\partial \dot{q}^{s}} \dot{\xi}^{s}(t) d t=\left.\frac{\partial L_{A}}{\partial \dot{q}^{s}} \xi^{s}\right|_{t_{1}} ^{t_{2}}-\int_{t_{1}}^{t_{2}} \xi^{s} \frac{d}{d t} \frac{\partial L_{A}}{\partial \dot{q}^{s}} d t \\
\int_{t_{1}}^{t_{2}} \frac{\partial L_{A}}{\partial A_{P}^{\alpha} q^{s}} A_{P}^{\alpha} \xi^{s}(t) d t=\left.K_{P^{*}}^{1-\alpha} \frac{\partial L_{A}}{\partial A_{P}^{\alpha} q^{s}} \xi^{s}(t)\right|_{t_{1}} ^{t_{2}}-\int_{t_{1}}^{t_{2}} \xi^{s}(t) B_{P^{*}}^{\alpha} \frac{\partial L_{A}}{\partial A_{P}^{\alpha} q^{s}} d t .
\end{gathered}
$$

Because $\xi^{s}\left(t_{1}\right)=\xi^{s}\left(t_{2}\right)=0$, we have

$$
\int_{t_{1}}^{t_{2}}\left(\frac{\partial L_{A}}{\partial q^{s}}-\frac{d}{d t} \frac{\partial L_{A}}{\partial \dot{q}^{s}}-B_{P^{*}}^{\alpha} \frac{\partial L_{A}}{\partial A_{P}^{\alpha} q^{s}}\right) \xi^{s}(t) d t=0 .
$$

We obtain (29) applying the fundamental lemma of the calculus of variations.

In order to prove a fractional Noether's theorem for functional (27) we adopt a technique used in [16], the proof is done in two steps: we begin by proving a Noether's theorem without transformation of the time (without transformation of the independent variable); then, using a technique of timere-parametrization, we obtain Noether's theorem in its general form.

Definition 4. Functional (27) is invariant under the $\epsilon$-parameter group of infinitesimal transformations

$$
\bar{q}^{s}(t)=q^{s}(t)+\epsilon \xi^{s}(t, q)
$$

if and only if

$$
\int_{t_{a}}^{t_{b}} L_{A}\left(t, q^{s}(t), \dot{q}^{s}(t), A_{P}^{\alpha} q^{s}(t)\right) d t=\int_{t_{a}}^{t_{b}} L_{A}\left(t, \bar{q}^{s}(t), \dot{\bar{q}}^{s}(t), A_{P}^{\alpha} \bar{q}^{s}(t)\right) d t
$$

for any subinterval $\left[t_{a}, t_{b}\right] \subseteq\left[t_{1}, t_{2}\right]$. 
Theorem 2. If the functional (27) is invariant under infinitesimal transformations (30), then

$$
\begin{gathered}
\partial_{2} L_{A}\left(t, q^{s}(t), \dot{q}^{s}(t), A_{P}^{\alpha} q^{s}(t)\right) \xi^{s}(t, q)+\partial_{3} L_{A}\left(t, q^{s}(t), \dot{q}^{s}(t), A_{P}^{\alpha} q^{s}(t)\right) \dot{\xi}^{s}(t, q) \\
+\partial_{4} L_{A}\left(t, q^{s}(t), \dot{q}^{s}(t), A_{P}^{\alpha} q^{s}(t)\right) A_{P}^{\alpha} \xi^{s}(t, q)=0 .
\end{gathered}
$$

Proof. The condition (31) is valid for any subinterval $\left[t_{a}, t_{b}\right] \subseteq\left[t_{1}, t_{2}\right]$. Differentiating this condition with respect to $\epsilon$, and substituting $\epsilon=0$, and using the definitions and properties of generalized derivative operators given in Section 2, we arrive to

$$
\begin{gathered}
0=\partial_{2} L_{A}\left(t, q^{s}(t), \dot{q}^{s}(t), A_{P}^{\alpha} q^{s}(t)\right) \xi^{s}(t, q)+\partial_{3} L_{A}\left(t, q^{s}(t), \dot{q}^{s}(t), A_{P}^{\alpha} q^{s}(t)\right) \dot{\xi}^{s}(t, q) \\
+\left.\partial_{4} L_{A}\left(t, q^{s}(t), \dot{q}^{s}(t), A_{P}^{\alpha} q^{s}(t)\right) \frac{d}{d \epsilon}\left[A_{P}^{\alpha} q^{s}(t)+\epsilon A_{P}^{\alpha} \xi^{s}(t, q)\right]\right|_{\epsilon=0}
\end{gathered}
$$

The expression (33) is equivalent to (32).

Remark. Using the Euler-Lagrange equation (29), the necessary condition of invariance (32) is equivalent to

$$
\begin{gathered}
\xi^{s}(t, q) \frac{d}{d t} \partial_{3} L_{A}\left(t, q^{s}(t), \dot{q}^{s}(t), A_{P}^{\alpha} q^{s}(t)\right)+\partial_{3} L_{A}\left(t, q^{s}(t), \dot{q}^{s}(t), A_{P}^{\alpha} q^{s}(t)\right) \dot{\xi}^{s}(t, q) \\
+\partial_{4} L_{A}\left(t, q^{s}(t), \dot{q}^{s}(t), A_{P}^{\alpha} q^{s}(t)\right) A_{P}^{\alpha} \xi^{s}(t, q) \\
+\xi^{s}(t, q) B_{P}^{\alpha} \partial_{4} L_{A}\left(t, q^{s}(t), \dot{q}^{s}(t), A_{P}^{\alpha} q^{s}(t)\right)=0
\end{gathered}
$$

Imitating the proof from paper [22], we can prove the following a new "transfer formula" for generalized fractional derivative operators.

Theorem 3. Consider functions $f, g \in C^{\infty}\left(\left[t_{1}, t_{2}\right] ; \mathbb{R}^{n}\right)$ and assume the following condition $(\mathcal{C})$ : the sequences $\left(g^{(k)} \cdot K_{P_{1}}^{k-\alpha} f\right)_{k \in \mathbb{N}^{*}},\left(g^{(k)} \cdot K_{P_{2}}^{k-\alpha} f\right)_{k \in \mathbb{N}^{*}}$, $\left(f^{(k)} \cdot K_{P_{1}}^{k-\alpha}\left(g-g\left(t_{1}\right)\right)\right)_{k \in \mathbb{N}^{*}}$ and $\left(f^{(k)} \cdot K_{P_{2}}^{k-\alpha}\left(g-g\left(t_{2}\right)\right)\right)_{k \in \mathbb{N}^{*}}$ converge uniformly to 0 on $\left[t_{1}, t_{2}\right]$. Then, the following relation holds:

$$
\begin{gathered}
g \cdot A_{P}^{\alpha} f+f \cdot B_{P^{*}}^{\alpha} g=\frac{d}{d t}\left(\sum_{r=0}^{\infty}\left(g^{(r)} \cdot\left((-1)^{r} p K_{P_{1}}^{r+1-\alpha} f+l K_{P_{2}}^{r+1-\alpha} f\right)\right)\right) \\
+\frac{d}{d t}\left(\sum_{r=0}^{\infty}\left(f^{(r)} \cdot\left((-1)^{r} l K_{P_{1}}^{r+1-\alpha}\left(g-g\left(t_{1}\right)\right)+p K_{P_{2}}^{r+1-\alpha}\left(g-g\left(t_{2}\right)\right)\right)\right)\right) .
\end{gathered}
$$

Theorem 4. (Fractional Noether's theorem without transformation of time) If the functional (27) is invariant under the one-parameter group of 
transformation (30), and functions $\xi^{s}(t, q)$ and $\partial_{4} L$ satisfy the condition $(\mathcal{C})$ of Theorem 3, then

$$
\begin{gathered}
\frac{d}{d t}\left(\xi^{s} \partial_{3} L_{A}+\sum_{r=0}^{\infty}\left(\left(\partial_{4} L_{A}\right)^{(r)} \cdot\left((-1)^{r} p K_{P_{1}}^{r+1-\alpha} \xi^{s}+l K_{P_{2}}^{r+1-\alpha} \xi^{s}\right)\right)\right) \\
+\frac{d}{d t}\left(\sum_{r=0}^{\infty}\left(\left(\xi^{s}\right)^{(r)} \cdot\left((-1)^{r} l K_{P_{1}}^{r+1-\alpha}\left(\partial_{4} L_{A}-\partial_{4} L_{A}\left(t_{1}\right)\right)\right)\right)\right) \\
+\frac{d}{d t}\left(\sum_{r=0}^{\infty}\left(\left(\xi^{s}\right)^{(r)} \cdot p K_{P_{2}}^{r+1-\alpha}\left(\partial_{4} L_{A}-\partial_{4} L_{A}\left(t_{2}\right)\right)\right)\right)=0
\end{gathered}
$$

along any fractional extremal with classical and generalized fractional derivatives $q^{s}(t), t \in\left[t_{1}, t_{2}\right]$.

Proof. We combine equation (34) and Theorem 3.

Next, we consider a more general notion of invariance for the integral functional (27).

Definition 5. (Invariance of (27)) The functional (27) is said to be invariant under the $\epsilon$-parameter group of infinitesimal transformations

$$
\begin{gathered}
\bar{t}=t+\epsilon \tau(t, q), \\
\bar{q}^{s}(t)=q^{s}(t)+\epsilon \xi^{s}(t, q),
\end{gathered}
$$

if and only if

$$
\int_{t_{a}}^{t_{b}} L_{A}\left(t, q^{s}(t), \dot{q}^{s}(t), A_{P}^{\alpha} q^{s}(t)\right) d t=\int_{\bar{t}_{a}}^{\bar{t}_{b}} L_{A}\left(\bar{t}, \bar{q}^{s}(\bar{t}), \dot{\bar{q}}^{s}(\bar{t}), A_{P}^{\alpha} \bar{q}^{s}(\bar{t})\right) d \bar{t}
$$

for any subinterval $\left[t_{a}, t_{b}\right] \subseteq\left[t_{1}, t_{2}\right]$.

Theorem 5. (Fractional Noether's theorem) If the functional (27) is invariant under the one-parameter group of transformations (37) and (38), and the functions $\xi^{s}(t, q)$ and $\partial_{4} L$ satisfy condition $(\mathcal{C})$ of Theorem 3, then

$$
\begin{gathered}
\frac{d}{d t}\left(\xi^{s} \partial_{3} L_{A}+\tau\left(L_{A}-\dot{q}^{s} \partial_{3} L_{A}-\alpha \partial_{4} L_{A} \cdot A_{P}^{\alpha} q^{s}\right)\right) \\
+\frac{d}{d t}\left(\sum_{r=0}^{\infty}\left(\left(\partial_{4} L_{A}\right)^{(r)} \cdot\left((-1)^{r} p K_{P_{1}}^{r+1-\alpha} \xi^{s}+l K_{P_{2}}^{r+1-\alpha} \xi^{s}\right)\right)\right) \\
+\frac{d}{d t}\left(\sum_{r=0}^{\infty}\left(\xi^{s}\right)^{(r)} \cdot\left((-1)^{r} l K_{P_{1}}^{r+1-\alpha}\left(\partial_{4} L_{A}-\partial_{4} L_{A}\left(t_{1}\right)\right)\right)\right)
\end{gathered}
$$




$$
+\frac{d}{d t}\left(\sum_{r=0}^{\infty}\left(\xi^{s}\right)^{(r)} \cdot\left(p K_{P_{2}}^{r+1-\alpha}\left(\partial_{4} L_{A}-\partial_{4} L_{A}\left(t_{2}\right)\right)\right)\right)=0 .
$$

along any fractional extremal with classical and generalized fractional derivatives $q^{s}(t), t \in\left[t_{1}, t_{2}\right]$.

Proof. Our proof is an extension of the method used in [36]. For that we re-parametrize the time (the independent variable $t$ ) by the Lipschitz transformation

$$
\left[t_{1}, t_{2}\right] \supseteq t \mapsto \sigma f(\lambda) \in\left[\sigma_{t_{1}}, \sigma_{t_{2}}\right]
$$

that satisfies

$$
t_{\sigma}^{\prime}=\frac{d t(\sigma)}{d \sigma}=f(\lambda)=1 \text { if } \lambda=0 .
$$

The functional (27) is reduced, in this way, to an autonomous functional:

$$
J\left[t(\cdot), q^{s}(t(\cdot))\right]=\int_{\sigma_{t_{1}}}^{\sigma_{t_{2}}} L\left(t(\sigma), q^{s}(t(\sigma)), \dot{q}^{s}(t(\sigma)), A_{P}^{\alpha} q^{s}(t(\sigma))\right) t_{\sigma}^{\prime} d \sigma
$$

where $t\left(\sigma_{t_{1}}\right)=t_{1}, t\left(\sigma_{t_{2}}\right)=t_{2}, t_{\sigma}^{\prime}$, and $\left(q_{\sigma}^{s}\right)^{\prime}=\frac{d q^{s}(t(\sigma))}{d \sigma}$. Using the definitions and properties of fractional derivatives given in Section 2, we have:

Case 1: If $P=P_{1}=\left\langle t_{1}, t, t_{2}, 1,0\right\rangle$,

$$
\begin{gathered}
A_{P_{1}}^{\alpha} q^{s}(t(\sigma))=\frac{1}{\Gamma(1-\alpha)} \frac{d}{d t(\sigma)} \int_{\frac{t_{1}}{f(\lambda)}}^{\sigma f(\lambda)}(\sigma f(\lambda)-\theta)^{-\alpha} q^{s}\left(\theta f^{-1}(\lambda)\right) d \theta \\
=\frac{\left(t_{\sigma}^{\prime}\right)^{-\alpha}}{\Gamma(1-\alpha)} \frac{d}{d \sigma} \int_{\frac{t_{1}}{\left(t_{\sigma}^{\prime}\right)^{2}}}^{\sigma}(\sigma-\rho)^{-\alpha} q^{s}(\rho) d \rho=\left(t_{\sigma}^{\prime}\right)^{-\alpha} \frac{t_{1}}{\left(t_{\sigma}^{\prime}\right)^{2}} D_{\sigma}^{\alpha} q^{s}(\sigma) .
\end{gathered}
$$

Case 2: If $P=P_{2}=\left\langle t_{1}, t, t_{2}, 0,1\right\rangle$,

$$
\begin{gathered}
A_{P_{2}}^{\alpha} q^{s}(t(\sigma))=\frac{1}{\Gamma(1-\alpha)}\left(-\frac{d}{d t(\sigma)}\right) \int_{\sigma f(\lambda)}^{\frac{t_{2}}{f(\lambda)}}(\theta-\sigma f(\lambda))^{-\alpha} q^{s}\left(\theta f^{-1}(\lambda)\right) d \theta \\
=\frac{\left(t_{\sigma}^{\prime}\right)^{-\alpha}}{\Gamma(1-\alpha)}\left(-\frac{d}{d \sigma}\right) \int_{\sigma}^{\frac{t_{2}}{\left(t_{\sigma}^{\prime}\right)^{2}}}(\rho-\sigma)^{-\alpha} q^{s}(\rho) d \rho=\left(t_{\sigma}^{\prime}\right)^{-\alpha}{ }_{\sigma} D_{\frac{t_{2}}{\left(t_{\sigma}^{\prime}\right)^{2}}} q^{s}(\sigma) .
\end{gathered}
$$

Case 3: If $P=P_{3}=\left\langle t_{1}, t, t_{2}, \frac{1}{2}, \frac{1}{2}\right\rangle$,

$$
A_{P_{3}}^{\alpha} q^{s}(t(\sigma))=\frac{1}{\Gamma(1-\alpha)} \frac{d}{d t(\sigma)} \int_{\frac{t_{1}}{f(\lambda)}}^{\frac{t_{2}}{f(\lambda)}}|\theta-\sigma f(\lambda)|^{-\alpha} q^{s}\left(\theta f^{-1}(\lambda)\right) d \theta
$$




$$
=\frac{\left(t_{\sigma}^{\prime}\right)^{-\alpha}}{\Gamma(1-\alpha)} \frac{d}{d \sigma} \int_{\frac{t_{1}}{\left(t_{\sigma}^{\prime}\right)^{2}}}^{\frac{t_{2}}{\left(t_{\sigma}^{\prime}\right)^{2}}}|\rho-\sigma|^{-\alpha} q^{s}(\rho) d \rho=\left(t_{\sigma}^{\prime}\right)^{-\alpha R_{t_{1}}} D_{\frac{t_{2}}{\left(t_{\sigma}^{\prime}\right)^{2}}}^{\alpha} q^{s}(\sigma) .
$$

Introducing the operator $A_{P_{\sigma}}^{\alpha}$, we have

$$
A_{P}^{\alpha} q^{s}(t(\sigma))=\left(t_{\sigma}^{\prime}\right)^{-\alpha} A_{P_{\sigma}}^{\alpha} q^{s}(\sigma)
$$

Then,

$$
\begin{gathered}
\bar{J}_{A}\left[t(\cdot), q^{s}(t(\cdot))\right]=\int_{\sigma_{t_{1}}}^{\sigma_{t_{2}}} L_{A}\left(t(\sigma), q^{s}(t(\sigma)), \frac{\left(q_{\sigma}^{s}\right)^{\prime}}{t_{\sigma}^{\prime}},\left(t_{\sigma}^{\prime}\right)^{-\alpha} A_{P_{\sigma}}^{\alpha} q^{s}(\sigma)\right) t_{\sigma}^{\prime} d \sigma \\
=\int_{\sigma_{t_{1}}}^{\sigma_{t_{2}}} \bar{L}_{A}\left(t(\sigma), q^{s}(t(\sigma)), t_{\sigma}^{\prime},\left(q_{\sigma}^{s}\right)^{\prime}, A_{P_{\sigma}}^{\alpha} q^{s}(\sigma)\right) d \sigma \\
=\int_{t_{1}}^{t_{2}} L_{A}\left(t, q^{s}(t), \dot{q}^{s}(t), A_{P}^{\alpha} q^{s}(t)\right) d t=J_{A}\left[q^{s}(\cdot)\right] .
\end{gathered}
$$

If the integral functional (27) is invariant in the sense of Definition 5, then the integral functional (42) is invariant in the sense of Definition 4. It follows from Theorem 4 that

$$
\begin{aligned}
\frac{d}{d t}\left(\tau \cdot \partial_{3} \bar{L}_{A}\right. & \left.+\xi^{s} \cdot \partial_{4} \bar{L}_{A}+\sum_{r=0}^{\infty}\left(\left(\partial_{4} L_{A}\right)^{(r)} \cdot\left((-1)^{r} p K_{P_{1}}^{r+1-\alpha} \xi^{s}+l K_{P_{2}}^{r+1-\alpha} \xi^{s}\right)\right)\right) \\
& +\frac{d}{d t}\left(\sum_{r=0}^{\infty}\left(\xi^{s}\right)^{(r)} \cdot\left((-1)^{r} l K_{P_{1}}^{r+1-\alpha}\left(\partial_{4} L_{A}-\partial_{4} L_{A}\left(t_{1}\right)\right)\right)\right) \\
& +\frac{d}{d t}\left(\sum_{r=0}^{\infty}\left(\xi^{s}\right)^{(r)} \cdot\left(p K_{P_{2}}^{r+1-\alpha}\left(\partial_{4} L_{A}-\partial_{4} L_{A}\left(t_{2}\right)\right)\right)\right)=0
\end{aligned}
$$

For $\lambda=0$, the condition (41) allows us to write that

$$
A_{P_{\sigma}}^{\alpha} q^{s}(t(\sigma))=A_{P}^{\alpha} q^{s}(t) .
$$

And, therefore, we get

$$
\partial_{4} \bar{L}_{A}=\partial_{3} L_{A}, \quad \partial_{5} \bar{L}_{A}=\partial_{4} L_{A},
$$

and

$$
\begin{gathered}
\frac{\partial \bar{L}_{A}}{\partial t_{\sigma}^{\prime}}=L_{A}+\partial_{3} \bar{L}_{A} \cdot t_{\sigma}^{\prime} \frac{\partial}{\partial t_{\sigma}^{\prime}} \frac{\left(q_{\sigma}^{s}\right)^{\prime}}{t_{\sigma}^{\prime}}+\partial_{5} \bar{L}_{A} \cdot \frac{\partial}{\partial t_{\sigma}^{\prime}}\left(\left(t_{\sigma}^{\prime}\right)^{-\alpha} A_{P_{\sigma}}^{\alpha} q^{s}(\sigma)\right) \cdot t_{\sigma}^{\prime} \\
=L_{A}-\dot{q}^{s} \partial_{3} L_{A}-\alpha \partial_{4} L_{A} \cdot A_{P}^{\alpha} q^{s}
\end{gathered}
$$


We obtain (40) substituting (44) and (45) into equation (43).

Second, we consider the problem of extremizing the functional as following

$$
J_{B}(q)=\int_{t_{1}}^{t_{2}} L_{B}\left(t, q^{s}(t), \dot{q}^{s}(t), B_{P}^{\alpha} q^{s}(t)\right) d t
$$

subject to the boundary conditions

$$
q^{s}\left(t_{1}\right)=q_{t_{1}}^{s}, \quad q^{s}\left(t_{2}\right)=q_{t_{2}}^{s},
$$

where $\left[t_{1}, t_{2}\right] \subset \mathbb{R}, t_{1}<t_{2}, 0<\alpha<1, s=1,2, \cdots, n$, the Lagrangian $L_{B}\left(t, q^{s}(t), \dot{q}^{s}(t), B_{P}^{\alpha} q^{s}(t)\right):\left(\left[t_{1}, t_{2}\right] \times \mathbb{R}^{n} \times \mathbb{R}^{n} \times \mathbb{R}^{n} ; \mathbb{R}\right)$ is assumed $C^{2}$-functions with respect to all its arguments. We denote by $\partial_{i} L_{B}$ the partial derivative of $L_{B}$ with respect to its $i$ th argument, $i=1,2, \cdots, 5$.

Using the same reasoning, we can obtain following results:

Theorem 6. Let $q^{s}(t)$ be a solution to problem (46)-(47). Then $q^{s}(t)$ satisfies the generalized Euler-Lagrange equation

$$
\frac{\partial L_{B}}{\partial q^{s}}-\frac{d}{d t} \frac{\partial L_{B}}{\partial \dot{q}^{s}}-A_{P^{*}}^{\alpha} \frac{\partial L_{B}}{\partial B_{P}^{\alpha} q^{s}}=0
$$

for $t \in\left[t_{1}, t_{2}\right]$.

Theorem 7. If the functional (46) is invariant under transformations (30), then

$$
\begin{gathered}
\partial_{2} L_{B}\left(t, q^{s}(t), \dot{q}^{s}(t), B_{P}^{\alpha} q^{s}(t)\right) \xi^{s}(t, q)+\partial_{3} L_{B}\left(t, q^{s}(t), \dot{q}^{s}(t), B_{P}^{\alpha} q^{s}(t)\right) \dot{\xi}^{s}(t, q) \\
+\partial_{4} L_{B}\left(t, q^{s}(t), \dot{q}^{s}(t), B_{P}^{\alpha} q^{s}(t)\right) B_{P}^{\alpha} \xi^{s}(t, q)=0 .
\end{gathered}
$$

Remark. Using the Euler-Lagrange equation (48), the necessary condition of invariance (49) is equivalent to

$$
\begin{gathered}
\xi^{s}(t, q) \frac{d}{d t} \partial_{3} L_{B}\left(t, q^{s}(t), \dot{q}^{s}(t), B_{P}^{\alpha} q^{s}(t)\right)+\partial_{3} L_{B}\left(t, q^{s}(t), \dot{q}^{s}(t), B_{P}^{\alpha} q^{s}(t)\right) \dot{\xi}^{s}(t, q) \\
+\partial_{4} L_{B}\left(t, q^{s}(t), \dot{q}^{s}(t), B_{P}^{\alpha} q^{s}(t)\right) B_{P}^{\alpha} \xi^{s}(t, q) \\
+\xi^{s}(t, q) A_{P^{*}}^{\alpha} \partial_{4} L_{B}\left(t, q^{s}(t), \dot{q}^{s}(t), B_{P}^{\alpha} q^{s}(t)\right)=0
\end{gathered}
$$

Theorem 8. Consider the functions $f, g \in C^{\infty}\left(\left[t_{1}, t_{2}\right] ; \mathbb{R}^{n}\right)$ and assume the following condition $(\mathcal{C})$ : the sequences $\left(g^{(k)} \cdot K_{P_{1}}^{k-\alpha}\left(f-f\left(t_{1}\right)\right)\right)_{k \in \mathbb{N}^{*}}$, $\left(g^{(k)} \cdot K_{P_{2}}^{k-\alpha}\left(f-f\left(t_{2}\right)\right)\right)_{k \in \mathbb{N}^{*}},\left(f^{(k)} \cdot K_{P_{1}}^{k-\alpha} g\right)_{k \in \mathbb{N}^{*}}$ and $\left(f^{(k)} \cdot K_{P_{2}}^{k-\alpha} g\right)_{k \in \mathbb{N}^{*}}$ converge uniformly to 0 on $\left[t_{1}, t_{2}\right]$. Then, the following relation holds: 


$$
\begin{gathered}
g \cdot B_{P}^{\alpha} f+f \cdot A_{P^{*}}^{\alpha} g=\frac{d}{d t}\left(\sum_{r=0}^{\infty}\left(f^{(r)} \cdot\left((-1)^{r} l K_{P_{1}}^{r+1-\alpha} g+p K_{P_{2}}^{r+1-\alpha} g\right)\right)\right) \\
+\frac{d}{d t}\left(\sum_{r=0}^{\infty}\left(g^{(r)} \cdot\left((-1)^{r} p K_{P_{1}}^{r+1-\alpha}\left(f-f\left(t_{1}\right)\right)+l K_{P_{2}}^{r+1-\alpha}\left(f-f\left(t_{2}\right)\right)\right)\right)\right) .
\end{gathered}
$$

Theorem 9. If the functional (46) is invariant under the one-parameter group of transformation (30), and the functions $\xi^{s}(t, q)$ and $\partial_{4} L$ satisfy the condition (C) of Theorem 8, then

$$
\begin{gathered}
\frac{d}{d t}\left(\xi^{s} \partial_{3} L_{B}+\sum_{r=0}^{\infty}\left(\left(\partial_{4} L_{B}\right)^{(r)} \cdot\left((-1)^{r} p K_{P_{1}}^{r+1-\alpha}\left(\xi^{s}-\xi^{s}\left(t_{1}\right)\right)+l K_{P_{2}}^{r+1-\alpha}\left(\xi^{s}-\xi^{s}\left(t_{2}\right)\right)\right)\right)\right) \\
+\frac{d}{d t}\left(\sum_{r=0}^{\infty}\left(\left(\xi^{s}\right)^{(r)} \cdot\left((-1)^{r} l K_{P_{1}}^{r+1-\alpha} \partial_{4} L_{B}+p K_{P_{2}}^{r+1-\alpha} \partial_{4} L_{B}\right)\right)\right)=0
\end{gathered}
$$

along any fractional extremal with classical and generalized fractional derivatives $q^{s}(t), t \in\left[t_{1}, t_{2}\right]$.

Theorem 10. If the functional (46) is invariant under the one-parameter group of transformations (37) and (38), and the functions $\xi^{s}(t, q)$ and $\partial_{4} L$ satisfy the condition $(\mathcal{C})$ of Theorem 8 , then

$$
\begin{gathered}
\frac{d}{d t}\left(\xi^{s} \partial_{3} L_{B}+\tau\left(L_{B}-\dot{q}^{s} \partial_{3} L_{B}-\alpha \partial_{4} L_{B} \cdot B_{P}^{\alpha} q^{s}\right)\right) \\
+\frac{d}{d t}\left(\sum_{r=0}^{\infty}\left(\left(\xi^{s}\right)^{(r)} \cdot\left((-1)^{r} l K_{P_{1}}^{r+1-\alpha} \partial_{4} L_{B}+p K_{P_{2}}^{r+1-\alpha} \partial_{4} L_{B}\right)\right)\right) \\
+\frac{d}{d t}\left(\sum_{r=0}^{\infty}\left(\left(\partial_{4} L_{B}\right)^{(r)} \cdot\left((-1)^{r} p K_{P_{1}}^{r+1-\alpha}\left(\xi^{s}-\xi^{s}\left(t_{1}\right)\right)+l K_{P_{2}}^{r+1-\alpha}\left(\xi^{s}-\xi^{s}\left(t_{2}\right)\right)\right)\right)\right)=0
\end{gathered}
$$

along any fractional extremal with classical and generalized fractional derivatives $q^{s}(t), t \in\left[t_{1}, t_{2}\right]$.

In particular, when parameters $p$ and $l$ take different values, we have following corollaries:

Corollary 1. If $P=P_{1}=\left\langle t_{1}, t, t_{2}, 1,0\right\rangle$, the functional $J_{1}(q)$

$$
J_{1}(q)=\int_{t_{1}}^{t_{2}} L_{1}\left(t, q^{s}(t), \dot{q}^{s}(t),{ }_{t_{1}} D_{t}^{\alpha} q^{s}(t)\right) d t
$$


is invariant under the one-parameter group of transformations (37) and (38), and functions $\xi^{s}(t, q)$ and $\partial_{4} L_{1}$ satisfy the condition $(\mathcal{C})$ of Theorem 3, then

$$
\begin{gathered}
\frac{d}{d t}\left(\xi^{s} \partial_{3} L_{1}+\tau\left(L_{1}-\dot{q}^{s} \partial_{3} L_{1}-\alpha \partial_{4} L_{1} \cdot{ }_{t_{1}} D_{t}^{\alpha} q^{s}\right)\right) \\
+\frac{d}{d t}\left(\sum_{r=0}^{\infty}\left((-1)^{r}\left(\partial_{4} L_{1}\right)^{(r)} \cdot{ }_{t_{1}} I_{t}^{r+1-\alpha} \xi^{s}+\left(\xi^{s}\right)^{(r)} \cdot{ }_{t} I_{t_{2}}^{r+1-\alpha}\left(\partial_{4} L_{1}-\partial_{4} L_{1}\left(t_{2}\right)\right)\right)\right)=0
\end{gathered}
$$

along any fractional extremal with classical and left Riemann-Liouville fractional derivatives $q^{s}(t), t \in\left[t_{1}, t_{2}\right]$.

This is the result of the paper [24].

Corollary 2. If $P=P_{1}=\left\langle t_{1}, t, t_{2}, 1,0\right\rangle$, the functional $J_{2}(q)$

$$
J_{2}(q)=\int_{t_{1}}^{t_{2}} L_{2}\left(t, q^{s}(t), \dot{q}^{s}(t),{ }_{t_{1}}^{C} D_{t}^{\alpha} q^{s}(t)\right) d t
$$

is invariant under the one-parameter group of transformations (37) and (38), and the functions $\xi^{s}(t, q)$ and $\partial_{4} L_{2}$ satisfy the condition $(\mathcal{C})$ of Theorem 8 , then

$$
\begin{gathered}
\frac{d}{d t}\left(\xi^{s} \partial_{3} L_{2}+\tau\left(L_{2}-\dot{q}^{s} \partial_{3} L_{2}-\alpha \partial_{4} L_{2} \cdot{ }_{t_{1}}^{C} D_{t}^{\alpha} q^{s}\right)\right) \\
\left.+\frac{d}{d t}\left(\sum_{r=0}^{\infty}\left((-1)^{r}\left(\partial_{4} L_{2}\right)^{(r)} \cdot{ }_{t_{1}} I_{t}^{r+1-\alpha}\left(\xi^{s}-\xi^{s}\left(t_{1}\right)\right)+\left(\xi^{s}\right)^{(r)} \cdot{ }_{t} I_{t_{2}}^{r+1-\alpha} \partial_{4} L_{2}\right)\right)\right)=0,
\end{gathered}
$$

along any fractional extremal with classical and left Caputo fractional derivatives $q^{s}(t), t \in\left[t_{1}, t_{2}\right]$.

The Noether theorem from paper [25] can be derived from Corollary 2.

Corollary 3. If $P=P_{2}=\left\langle t_{1}, t, t_{2}, 0,1\right\rangle$, the functional $J_{3}(q)$

$$
J_{3}(q)=\int_{t_{1}}^{t_{2}} L_{3}\left(t, q^{s}(t), \dot{q}^{s}(t),{ }_{t} D_{t_{2}}^{\alpha} q^{s}(t)\right) d t
$$

is invariant under the one-parameter group of transformations (37) and (38), and the functions $\xi^{s}(t, q)$ and $\partial_{4} L_{3}$ satisfy the condition $(\mathcal{C})$ of Theorem 3 , then

$$
\begin{gathered}
\frac{d}{d t}\left(\xi^{s} \partial_{3} L_{3}+\tau\left(L_{2}-\dot{q}^{s} \partial_{3} L_{3}+\alpha \partial_{4} L_{3} \cdot{ }_{t} D_{t_{2}}^{\alpha} q^{s}\right)\right) \\
+\frac{d}{d t}\left(\sum_{r=0}^{\infty}\left(\left(\partial_{4} L_{3}\right)^{(r)} \cdot{ }_{t} I_{t_{2}}^{r+1-\alpha} \xi^{s}+(-1)^{r}\left(\xi^{s}\right)^{(r)} \cdot{ }_{t_{1}} I_{t}^{r+1-\alpha}\left(\partial_{4} L_{3}-\partial_{4} L_{3}\left(t_{1}\right)\right)\right)\right)=0
\end{gathered}
$$


along any fractional extremal with classical and right Riemann-Liouville fractional derivatives $q^{s}(t), t \in\left[t_{1}, t_{2}\right]$.

Corollary 4. If $P=P_{1}=\left\langle t_{1}, t, t_{2}, 1,0\right\rangle$, the functional $J_{4}(q)$

$$
J_{4}(q)=\int_{t_{1}}^{t_{2}} L_{4}\left(t, q^{s}(t), \dot{q}^{s}(t),{ }_{t}^{C} D_{t_{2}}^{\alpha} q^{s}(t)\right) d t
$$

is invariant under the one-parameter group of transformations (37) and (38), and the functions $\xi^{s}(t, q)$ and $\partial_{4} L_{2}$ satisfy the condition $(\mathcal{C})$ of Theorem 8 , then

$$
\begin{gathered}
\frac{d}{d t}\left(\xi^{s} \partial_{3} L_{4}+\tau\left(L_{4}-\dot{q}^{s} \partial_{3} L_{4}+\alpha \partial_{4} L_{4} \cdot{ }_{t}^{C} D_{t_{2}}^{\alpha} q^{s}\right)\right) \\
+\frac{d}{d t}\left(\sum_{r=0}^{\infty}\left(\left(\partial_{4} L_{4}\right)^{(r)} \cdot{ }_{t} I_{t_{2}}^{r+1-\alpha}\left(\xi^{s}-\xi^{s}\left(t_{2}\right)\right)+(-1)^{r}\left(\xi^{s}\right)^{(r)} \cdot{ }_{t_{1}} I_{t}^{r+1-\alpha} \partial_{4} L_{4}\right)\right)=0,
\end{gathered}
$$

along any fractional extremal with classical and right Caputo fractional derivatives $q^{s}(t), t \in\left[t_{1}, t_{2}\right]$.

Corollary 5. If $P=P_{3}=\left\langle t_{1}, t, t_{2}, \frac{1}{2}, \frac{1}{2}\right\rangle$, the functional $J_{5}(q)$

$$
J_{5}(q)=\int_{t_{1}}^{t_{2}} L_{5}\left(t, q^{s}(t), \dot{q}^{s}(t),{ }_{t_{1}}^{R} D_{t_{2}}^{\alpha} q^{s}(t)\right) d t
$$

is invariant under the one-parameter group of transformations (37) and (38), and the functions $\xi^{s}(t, q)$ and $\partial_{4} L_{5}$ satisfy the condition $(\mathcal{C})$ of Theorem 3 , then

$$
\begin{gathered}
\frac{d}{d t}\left(\xi^{s} \partial_{3} L_{5}+\tau\left(L_{5}-\dot{q}^{s} \partial_{3} L_{5}-\alpha \partial_{4} L_{5} \cdot{ }_{t_{1}}^{R} D_{t_{2}}^{\alpha} q^{s}\right)\right) \\
+\frac{1}{2} \frac{d}{d t}\left(\sum_{r=0}^{\infty}\left(\left(\partial_{4} L_{5}\right)^{(r)} \cdot\left((-1)^{r}{ }_{t_{1}} I_{t}^{r+1-\alpha} \xi^{s}+{ }_{t} I_{t_{2}}^{r+1-\alpha} \xi^{s}\right)\right)\right) \\
+\frac{1}{2} \frac{d}{d t}\left(\sum_{r=0}^{\infty}\left(\left(\xi^{s}\right)^{(r)} \cdot\left((-1)^{r}{ }_{t} I_{t}^{r+1-\alpha}\left(\partial_{4} L_{5}-\partial_{4} L_{5}\left(t_{1}\right)\right)\right)\right)\right. \\
+\frac{1}{2} \frac{d}{d t}\left(\sum_{r=0}^{\infty}\left(\left(\xi^{s}\right)^{(r)} \cdot{ }_{t} I_{t_{2}}^{r+1-\alpha}\left(\partial_{4} L_{5}-\partial_{4} L_{5}\left(t_{2}\right)\right)\right)\right)=0 .
\end{gathered}
$$

along any fractional extremal with classical and Riesz fractional derivatives $q^{s}(t), t \in\left[t_{1}, t_{2}\right]$.

Corollary 6. If $P=P_{3}=\left\langle t_{1}, t, t_{2}, \frac{1}{2}, \frac{1}{2}\right\rangle$, the functional $J_{6}(q)$

$$
J_{6}(q)=\int_{t_{1}}^{t_{2}} L_{6}\left(t, q^{s}(t), \dot{q}^{s}(t),{ }_{t_{1}}^{R C} D_{t_{2}}^{\alpha} q^{s}(t)\right) d t
$$


is invariant under the one-parameter group of transformations (37) and (38), and the functions $\xi^{s}(t, q)$ and $\partial_{4} L_{6}$ satisfy the condition $(\mathcal{C})$ of Theorem 8, then

$$
\begin{gathered}
\frac{d}{d t}\left(\xi^{s} \partial_{3} L_{6}+\tau\left(L_{6}-\dot{q}^{s} \partial_{3} L_{6}-\alpha \partial_{4} L_{6} \cdot{ }_{t_{1}}^{R C} D_{t_{2}}^{\alpha} q^{s}\right)\right) \\
+\frac{1}{2} \frac{d}{d t}\left(\sum_{r=0}^{\infty}\left(\left(\partial_{4} L_{6}\right)^{(r)} \cdot\left((-1)^{r}{ }_{t_{1}} I_{t}^{r+1-\alpha}\left(\xi^{s}-\xi^{s}\left(t_{1}\right)\right)+{ }_{t} I_{t_{2}}^{r+1-\alpha}\left(\xi^{s}-\xi\left(t_{2}\right)\right)\right)\right)\right) \\
+\frac{1}{2} \frac{d}{d t}\left(\sum_{r=0}^{\infty}\left(\left(\xi^{s}\right)^{(r)} \cdot\left((-1)^{r}{ }_{t_{1}} I_{t}^{r+1-\alpha} \partial_{4} L_{6}+{ }_{t} I_{t_{2}}^{r+1-\alpha} \partial_{4} L_{6}\right)\right)\right)=0
\end{gathered}
$$

along any fractional extremal with classical and Riesz-Caputo fractional derivatives $q^{s}(t), t \in\left[t_{1}, t_{2}\right]$.

\section{Conclusion}

Almost all classical processes observed in the physical world are nonconservative. The fractional mathematical model can more accurately describe the dynamics of the systems. Over the past 20 years,studies on fractional variational problems made a number of important accomplishments. In this paper, we study generalized fractional variation problems, and the main results are: (1) Two new "transfer formulas" about generalized fractional derivative operators; (2) A extension of Noether's theorem to fractional problems of the calculus of variations with classical and generalized fractional derivative operators. It provides an explicit conservation law. The formula given is algorithmic, it can be used to computer conserved quantity to arbitrary high order approximations. Nevertheless, the conservation law is only given as a series of function: in most cases, it is not easily computable. However, an arbitrary closed approximation of this quantity can be obtained with a truncation. The results may be further applied to the area of fractional optimal control in future.

\section{Acknowledgments}

This work has been supported by the National Natural Science Foundation of China under Grant Nos. 10872037, 11472063 and the Natural Science Foundation of Anhui Province under Grant No. 070416226. The authors are very grateful to referees for their valuable comments and helpful suggestions. 


\section{References}

[1] A.E. Noether, Invariant variations problem, Nachr. Akad. Wiss. Göttingen. Math. Phys. Ki., II (1918), 235-257.

[2] P.S. Bauer, Dissipative dynamical systems, Proc. Natl. Acad. Sci. USA, 17 (1931), 311-314.

[3] B.D. Vujanovic, S.E. Jones, Variational Methods in Non-conservative Phenomeana, Academic Press, San Diego (1989).

[4] F. Riewe, Nonconservative Lagrangian and Hamiltonian mechanics, Phys. Rev. E, 53 (1996), 1890-1899.

[5] F. Riewe, Mechanics with fractional derivatives, Phys. Rev. E, 55 (1997), 3581-3592.

[6] A.B. Malinowska, D.F.M. Torres, Introduction to the Fractional Calculus of Variations, Imperial Colloge Press, London - World Scientific Publishing, Singapore (2012).

[7] O.P. Agrawal, A new Lagrangian and a new Lagrange equation of motion for fractionally damped systems, J. Appl. Mech., 68 (2001), 339-341.

[8] O.P. Agrawal, Formulation of Euler-Lagrange Equations for fractional variational problems, J. Math. Anal. Appl., 272 (2002),368-379.

[9] O.P. Agrawal, Fractional variational calculus and the transversality conditions, J. Phys. A: Math. Theor., 39 (2006), 10375-10384.

[10] O.P. Agrawal, Fractional variational calculus in terms of Riesz fractional derivatives, J. Phys. A: Math. Theor., 40 (2007), 6287-6303.

[11] M. Klimek, Fractional sequential mechanics-models with symmetric fractional derivative, Czech. J. Phys., 51 (2001), 1348-1354.

[12] M. Klimek, Stationarity-conservation laws for fractional differential equations with variable coefficients, J. Phys A: Math. Gen., 35 (2002), 6675-93.

[13] D.W. Dreisigmeyer, P.M. Young, Nonconservative Lagrangian mechanics: A generalized function approach, J. Phys. A: Math. Gen., 36 (2003), 82978310 .

[14] D.W. Dreisigmeyer, P.M. Young, Extending Bauer's corollary to fractional derivatives, J. Phys. A: Math. Gen., 37 (2003), L117-21. 
[15] J. Cresson, Fractional embedding of differential operators and Lagrangian systems, J. Math. Phys., 48 (2007), 033504.

[16] G.S.F. Frederico, D.F.M. Torres, A formulation of Noether's theorem for fractional problems of the calculus of variations, J. Math. Anal. Appl., 334 (2007), 834-846.

[17] G.S.F. Frederico, D.F.M. Torres, Fractional optimal control in the sense of Caputo and the fractional Noether's theorem, Int. Math. Forum, 3 (2008), 479-493.

[18] G.S.F. Frederico, D.F.M. Torres, Fractional Noether's theorem in the Riesz-Caputo sense, Appl. Math. Comput., 217 (2010), 1023-1033.

[19] S. Zhou, H. Fu, J.L. Fu, Symmetry theories of Hamiltonian systems with fractional derivatives. Sci. China Phys. Mech. Astron., 54 (2011), 18471853.

[20] T.M. Atanacković, S. Konjik, S.Pilipovic, S. Simic, Variational problems with fractional derivatives: invariance conditions and Noether's theorem. Nonlinear Anal., 71 (2009), 1504-1517.

[21] Z.X. Long, Y. Zhang, Noether's theorem for fractional variational problem from EI-Nabulsi extended exponentially fractional integral in phase space, Acta. Mech., 225 (2014), 77-90.

[22] L. Bourdin, J. Cresson, I. Greff, A continuous/ discrete fractional Noether's theorem, Commun. Nonlinear Sci. Numer. Simulat., 18 (2013), 878-887.

[23] O.P. Agrawal, Generalized variational problems and Euler-Lagrange equations, Computers and Mathematics with Applications, 59 (2010), 18521864.

[24] G.S.F. Frederico, D.F.M. Torres, Fractional Noether's theorem with classical and Riemann-Liouville derivatives, arXiv: 1209.1330v1[math.OC], 6 Sept. 2012.

[25] L. Bourdin, A class of fractional optimal control problems and fractional Pontryagin's systems. Existence of a fractional Noether's theorem, arXiv: 1203.1422v1[math.OC], 7 March 2012.

[26] D. Baleanu, K. Diethelm, E. Scalas, J.J. Trujillo, Fractional Calculus: Models and Numerical Methods, World Scientific Publishing, Singapore (2012). 
[27] R. Hilfer, Applications of Fractional Calculus in Physics, World Scientific, Singapore (2000).

[28] I. Podlubny, Fractional Differential Equations, Academic Press, San Diego, CA (1999).

[29] S.G. Samko, A.A. Kilbas, O.I. Marichev, Fractional Integrals and Derivatives, Translated and updated from the 1987 Russian original, Gordon and Breach, Yverdon (1993).

[30] A.A. Kilbas, H.M. Srivastava, J.J. Trujillo, Theory and Applications of Fractional Differential Equations, Elsevier, Amsterdam (2006).

[31] K.S. Miller, B. Ross, An Introduction to the Fractional Calculus and Fractional Differential Equations, Wiley, New York (1993).

[32] M.D. Ortigueira, Fractional Calculus for Scientists and Engineers, Lecture Notes in Electrical Engineering \# 84, Springer, Dordrecht (2011).

[33] R. Herrmann, Fractional Calculus - An Introduction for Physicists, World Scientific Publishing, Singapore (2011).

[34] C.P. Li, F.H. Zeng, Numerical Methods for Fractional Calculus, CRC Press (2015).

[35] R. Almeida, S. Pooseh, D.F.M. Torres, Computational Methods in the Fractional Calculus of Variations, Imperial College Press, London (2015).

[36] J. Jost, X. Li-Jost, Calculus of Variations, Cambridge University Press, Cambridge (1998). 